\title{
Kwashiorkor e distúrbio de coagulação - apresentação atípica de fibrose cística
}

\author{
Kwashiorkor and coagulation disturbance - atypical presentation of cystic fibrosis
}

\author{
Michelle de Oliveira T. Sundell ${ }^{1}$, Daniela Góis Meneses ${ }^{1}$, Antônio Fernando Ribeiro ${ }^{2}$, Elizete Aparecida L. C. Pinto ${ }^{2}$, Gabriel Hessel ${ }^{3}$
}

\section{RESUMO}

Objetivo: Enfatizar a apresentação clínica precoce da fibrose cística (FC) em lactente com Kwashiorkor e distúrbio de coagulação, decorrente de hipovitaminose $\mathrm{K}$.

Descrição do caso: Paciente com três meses e meio, sexo feminino, nascida a termo, peso de $2655 \mathrm{~g}$, estatura de $46 \mathrm{~cm}$, foi encaminhada para investigação de lesões perineais associadas à monilíase de difícil controle, refratária a diversos antifúngicos e corticoides. Quadro geral caracterizado por baixo ganho ponderal, edema e diarreia. Admissão hospitalar para investigação com hipótese diagnóstica de Kwashiorkor de origem primária ou secundária. Paciente mantida em aleitamento materno exclusivo, sendo observadas perda ponderal e persistência da diarreia. Na internação, foi iniciado tratamento de infecção do trato urinário. A paciente evoluiu com hemorragia digestiva alta e sangramento pela flebotomia em safena direita, sendo identificada coagulopatia responsiva à vitamina $\mathrm{K}$ e plasma fresco congelado. Na evolução, foi confirmada esteatorreia e hipoalbuminemia; as sorologias para sífilis, toxoplasmose, mononucleose, citomegalovírus, rubéola, HIV e hepatite B, apresentaram resultado negativo e a pesquisa da mutação $\Delta \mathrm{F} 508$ heterozigoto para FC foi positiva. A paciente apresentou piora do estado geral com sinais de sepse, evoluindo para óbito. O laudo necroscópico evidenciou elementos característicos de choque séptico com infecção pulmonar, sinais acentuados de desnutrição e fibrose cística do pâncreas.
Comentários: A FC pode manifestar-se com quadro de Kwashiorkor e distúrbio de coagulação por deficiência de vitamina K. Os profissionais de saúde devem estar atentos à possibilidade de FC no diagnóstico diferencial dessa situação.

Palavras-chave: fibrose cística; Kwashiorkor; vitamina $\mathrm{K}$; desnutrição.

\section{ABSTRACT}

Objective: To address the clinical presentation of cystic fibrosis (CF) in an infant presenting Kwashiorkor along with coagulation disturbance due to vitamin K deficiency.

Case description: A female baby aged three and a half months, born at term, with birth weight of $2655 \mathrm{~g}$, and height of $46 \mathrm{~cm}$, was referred to a university center due to perineal moniliasis refractory to therapy, including antifungal drugs and corticosteroids. She had poor weight gain, edema, and diarrhea. After hospital admission under the diagnostic hypothesis of Kwashiorkor of primary or secondary origin, the child received exclusive breastfeeding, but lost weight and maintained the diarrhea. At admission, a urinary tract infection was detected and treated. The child developed bleeding of upper digestive tract and phlebotomy incision at the right saphenous vein treated with vitamin $\mathrm{K}$ and fresh frozen plasma. Laboratory exams showed steatorrhea and hypoalbuminemia. Serology was negative for
Instituição: Departamento de Pediatria da Faculdade de Ciências Médicas da Universidade Estadual de Campinas (Unicamp), Campinas, SP, Brasil ${ }^{1}$ Mestre em Saúde da Criança e do Adolescente pela Faculdade de Ciências Médicas da Unicamp, Campinas, SP, Brasil

2Professor Doutor do Departamento de Pediatria da Faculdade de Ciências Médicas da Unicamp, Campinas, SP, Brasil

${ }^{3}$ Livre-docente em Pediatria pela Faculdade de Ciências Médicas da Unicamp; Professor Associado do Departamento de Pediatria da Faculdade de Ciências Médicas da Unicamp, Campinas, SP, Brasil

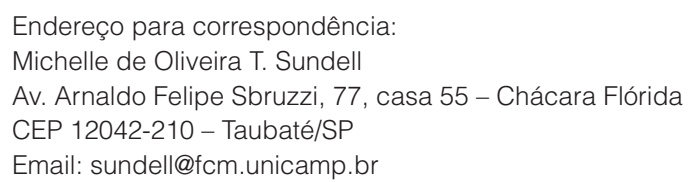

Endereço para correspondência:

Michelle de Oliveira T. Sundell

Av. Arnaldo Felipe Sbruzzi, 77, casa 55 - Chácara Flórida

CEP 12042-210 - Taubaté/SP

Email: sundell@fcm.unicamp.br

Fonte financiadora: Coordenação de Aperfeiçoamento de Pessoal de Nível Superior (Capes)

Conflito de interesse: nada a declarar

Recebido em: 2/5/2011

Aprovado em: 15/8/2011 
syphilis, toxoplasmosis, mononucleosis, cytomegalovirus, rubella, HIV and hepatitis $\mathrm{B}$. Heterozygous $\triangle \mathrm{F} 508$ mutation for $\mathrm{CF}$ was positive. The patient died with a septic shock. Necropsy showed that the septic shock had a pulmonary origin and that malnutrition was secondary to cystic fibrosis of pancreas.

Comments: CF may have a clinical presentation as Kwashiorkor with coagulation disturbance caused by vitamin $\mathrm{K}$ deficiency. Health professionals should be aware of this possibility in the differential diagnosis of infants with severe malnutrition and edema.

Key-words: cystic fibrosis; Kwashiorkor; vitamin K; malnutrition.

\section{Introdução}

A fibrose cística (FC) é uma doença autossômica recessiva. É considerada a doença hereditária letal mais comum em caucasianos, com uma estimativa de incidência de 1:2.000 a 1:3.000 nascimentos, sendo que um indivíduo em cada 25 é portador assintomático de uma mutação para o gene da FC. É mais rara na raça negra $(1: 17.000)$ e entre os asiáticos $(1: 90.000)^{(1,2)}$. É uma exocrinopatia caracterizada por secreções muito espessas e viscosas, acometendo principalmente o aparelho respiratório e o sistema digestório. As principais manifestações clínicas apresentadas nos casos clássicos de FC são: íleo meconial, lactente chiador, doença pulmonar obstrutiva crônica, pneumonias de repetição, diarreia crônica e desnutrição calórico-proteica ${ }^{(3)}$. Outra forma de apresentação clínica grave é a hipoproteinemia do lactente, na qual há edema, anemia e evolução grave, podendo ocorrer em 8 a $10 \%$ dos pacientes ${ }^{(4)}$.

Como variantes, há poucos relatos na literatura de lactentes jovens com hipoproteinemia e coagulopatia. Mérelle et $a^{(5)}$ descreveram uma paciente que apresentou equimoses a partir de dois meses de idade e desenvolveu hemorragia cerebral com dois meses e meio, sendo necessária uma intervenção cirúrgica; ainda apresentava baixo ganho ponderal e contava história de um primo com FC. Foi efetuado diagnóstico de deficiência de vitamina $\mathrm{K}$ e tratada com fatores de coagulação, transfusão sanguínea e vitamina $\mathrm{K}$ intramuscular. O diagnóstico de FC foi confirmado pelo teste do suor e a paciente apresentou boa evolução. Mei-Zahav et a ${ }^{(6)}$ relataram um caso de um menino que nasceu com $2680 \mathrm{~g}$ e desenvolveu desnutrição grave e doença respiratória. Com dois meses e meio apresentava peso abaixo do percentil 3, edema, hipoalbuminemia e esteatorreia. Fez-se exame de eletrólitos no suor e o resultado do cloro foi acima de $60 \mathrm{mmol} / \mathrm{L}$ em três amostras. Iniciou-se o tratamento com reposição de enzimas, vitaminas e zinco e o paciente apresentou melhora. Com 26 meses de idade, o peso e altura situavam-se entre os percentis 10 e 25 .

Este relato de caso teve como objetivo enfatizar a apresentação clínica precoce de Kwashiorkor em lactente com FC, juntamente com distúrbio de coagulação causado por deficiência de vitamina K. A publicação desse relato foi aprovada pelo Comitê de Ética em Pesquisa da Faculdade de Ciências Médicas da Universidade Estadual de Campinas (Unicamp).

\section{Descrição do caso}

Paciente com três meses e meio, gênero feminino, filha de pais não consanguíneos, encaminhada ao serviço por lesões cutâneas no períneo. Apresentou monilíase oral de difícil tratamento desde o $11^{\circ}$ dia de vida e, após 15 dias, também mostrou monilíase perineal, com piora progressiva do aspecto e extensão para a região proximal dos membros inferiores. $\mathrm{O}$ tratamento tópico com diversos antifúngicos e corticoides não resultou em melhora clínica. A mãe referiu baixo ganho ponderal, mesmo em vigência de aleitamento materno exclusivo, presença de episódios de diarreia e informava cristalização do suor na face.

Nascida de parto cesárea por sofrimento fetal agudo, a termo, com idade gestacional estimada em 40 semanas e cinco dias pelo método de Capurro, com peso de $2655 \mathrm{~g}$ e $46 \mathrm{~cm}$ de comprimento e Apgar 4/10. A paciente também apresentou icterícia precoce e necessitou de fototerapia durante dez dias. A mãe referia palidez e episódios frequentes de regurgitação; negou internações (exceto no período neonatal), transfusões sanguíneas ou cirurgias prévias. O desenvolvimento neuropsicomotor era adequado e a vacinação estava em dia. Foi negado antecedente familiar conhecido de FC. Na dinâmica familiar, a mãe referiu separação conjugal durante a gravidez.

Ao exame físico, a paciente apresentava-se com peso de 3360g (peso/idade < percentil 3 na curva de Tanner e Whitehouse $\left.^{(7)}\right)$, comprimento de $50 \mathrm{~cm}$ (altura/idade $<$ percentil 3), perímetro cefálico de $36 \mathrm{~cm}$ (perímetro cefálico/ idade no percentil 3), edema generalizado, monilíase oral e perineal, mucosas levemente ressecadas, sem alterações cardiopulmonares, sem visceromegalias.

Assim, procedeu-se à internação para investigar hipótese de desnutrição calórico-proteica de $3^{\circ}$ grau, tipo Kwashiorkor de origem primária ou secundária. As possíveis causas de Kwashiorkor secundário investigadas foram: FC, alergia à 
proteína do leite de vaca, síndrome de Shwachman-Diamond, deficiência de enteroquinase, deficiência de tripsinogênio e linfangiectasia intestinal. A criança foi mantida em aleitamento materno exclusivo, sendo observada perda ponderal, além de persistência dos episódios diarreicos (cinco a seis evacuações semilíquidas/dia).

O exame de urina tipo 1 revelou 1.920 .000 leucócitos/mL, leucócito-esterase positivo, nitritos negativos e urocultura $>10^{5}$ bactérias $/ \mathrm{mL}$ de Escherichia coli. Os demais exames laboratoriais estão demonstrados na Tabela 1.

No $4^{\circ}$ dia de internação, foi iniciado tratamento com amicacina devido à infecção do trato urinário. No mesmo dia, a paciente apresentou um episódio de hemorragia digestiva alta de moderado volume, melena e sangramento pela flebotomia em safena direita, com queda de hemoglobina de $7,0 \mathrm{~g} / \mathrm{dL}$ para $4,7 \mathrm{~g} / \mathrm{dL}$. O coagulograma mostrou índice internacional normalizado (RNI) de 10,2 (normal até 1,25), R de 2,06 (normal até 1,3 ) e contagem de plaquetas de $376.000 / \mathrm{mm}^{3}$. Administrou-se vitamina $\mathrm{K}$ e plasma fresco congelado, sem ocorrência de novos episódios de sangramento e melhora significativa dos exames de coagulação no $6^{\circ}$ dia de internação, mesmo após suspensão do plasma (RNI 1,29 e R 1,17).

Após estabilização clínica e resolução da coagulopatia, foi reiniciada a investigação com realização das sorologias para sífilis, toxoplasmose, mononucleose, citomegalovírus, rubéola, HIV e hepatite $\mathrm{B}$, todas com resultado negativo. $\mathrm{O}$ esteatócrito foi de 15,7 e 17,6\% (normal até $11 \%$ ) e o estudo genético evidenciou um alelo com a mutação $\Delta$ F508. Não foi realizado o teste do suor devido à instabilidade clínica inicial e evolução para anasarca, o que poderia prejudicar a interpretação do exame. A ultrassonografia abdominal revelou presença discreta de líquido livre em cavidade, sem linfonodomegalias ou alterações viscerais.

Diante da esteatorreia, sendo uma das hipóteses a alergia à proteína do leite de vaca, optou-se por introduzir o hidrolisado proteico e suspender o leite materno. $\mathrm{Na}$ evolução, houve piora da anasarca (ganho de $1 \mathrm{~kg}$ em sete dias) e manutenção de baixos níveis séricos de albumina (2,1g/dL), sendo iniciada a reposição de albumina intravenosa. O nível sérico de zinco foi de $52 \mu \mathrm{g} / \mathrm{dL}$ (normal de $70-150 \mu \mathrm{g} / \mathrm{dL}$ ), efetuando-se também a reposição de zinco e polivitamínicos.

No $12^{\circ}$ dia de internação hospitalar, a paciente apresentou hipoatividade, episódios de hipotermia, piora do edema, piora da tosse iniciada no $6^{\circ}$ dia de internação, insuficiência respiratória, oligúria e plaquetopenia, evoluindo para óbito. A necropsia evidenciou: sinais de choque, hidrotórax bilateral, hidropericárdio leve, ascite e erosões agudas em mucosa gástrica. Pulmões e vias aéreas: focos de broncopneumonia incipiente, rolhas de secreção mucosa na luz bronquiolar e moderado infiltrado inflamatório linfoplasmocitário peribronquiolar (Figura 1). Pâncreas com fibrose intra e interlobular difusa, ductos lobulares dilatados formando microcistos (Figura 2); intestino com hipotrofia das vilosidades. No diagnóstico final, a causa de óbito foi choque séptico com foco de origem pulmonar e a doença básica foi descrita como desnutrição acentuada secundária a má absorção, devido à FC do pâncreas.

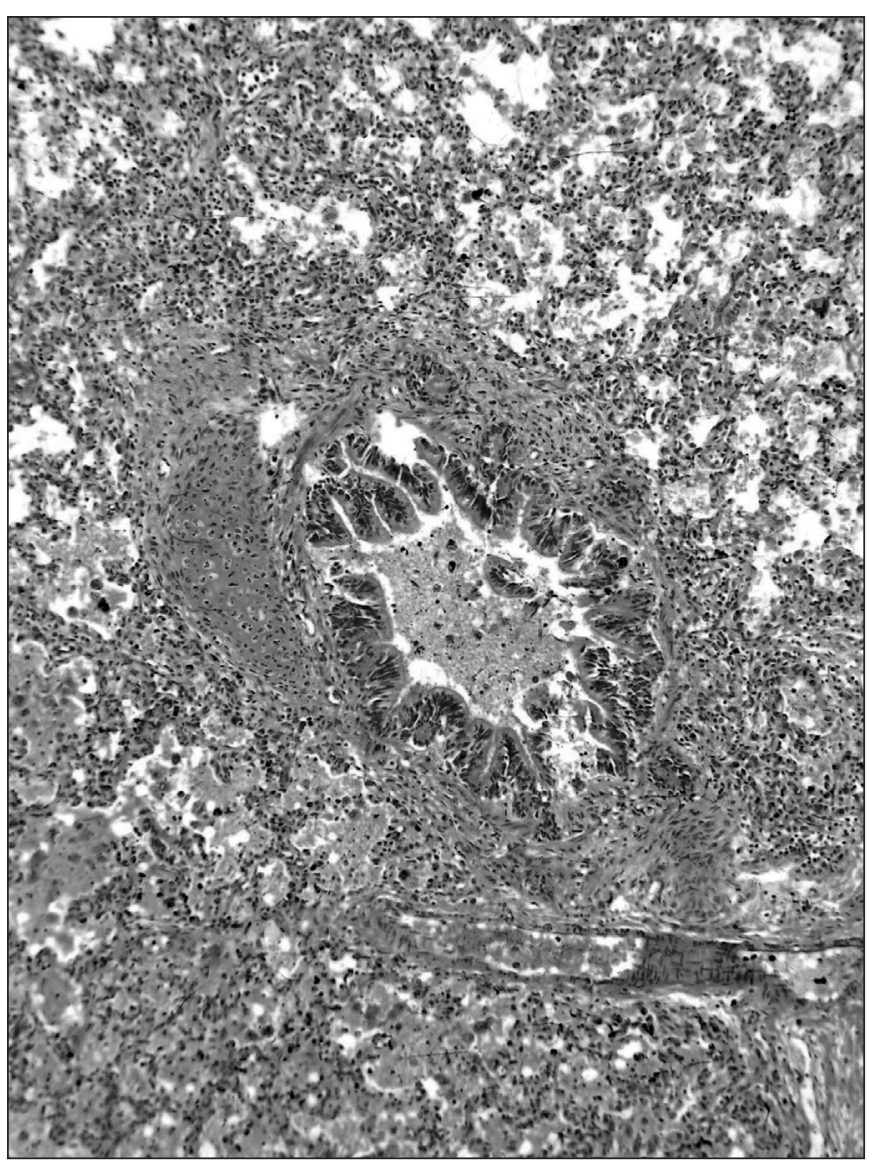

Figura 1 - Pulmão com presença de infiltrado inflamatório linfoplasmocitário peribronquiolar, rolhas de secreção mucosa na luz bronquiolar e exsudato inflamatório intra-alveolar (HE 40X)

Tabela 1 - Resultados de proteinograma e hematológicos

\begin{tabular}{lcc}
\hline $\begin{array}{l}\text { Exames } \\
\text { laboratoriais }\end{array}$ & Resultados & $\begin{array}{c}\text { Valor de } \\
\text { referência }\end{array}$ \\
\hline Proteínas totais $(\mathrm{g} / \mathrm{dL})$ & 3,50 & $6,6-8,7$ \\
Albumina $(\mathrm{g} / \mathrm{dL})$ & 1,78 & $3,2-5,4$ \\
Gama-globulina $(\mathrm{g} / \mathrm{dL})$ & 0,46 & $0,9-2,1$ \\
Hemoglobina $(\mathrm{g} / \mathrm{dL})$ & 7,00 & $12,0-15,0$ \\
Leucócitos $\left(\mathrm{mm}^{3}\right)$ & 17.650 & $4.000-10.000$ \\
Plaquetas $\left(\mathrm{mm}^{3}\right)$ & 315.000 & $150.000-400.000$ \\
\hline
\end{tabular}




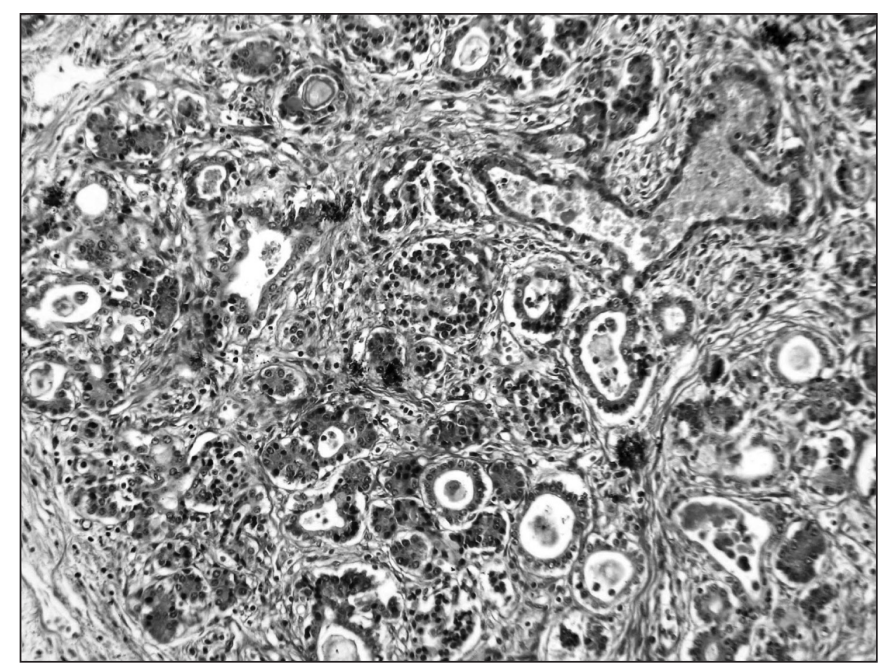

Figura 2 - Pâncreas com fibrose intra e interlobular; atrofia acinar e ductos dilatados formando microcistos (coloração de tricrômio de Masson 80X)

\section{Discussão}

A FC pode apresentar manifestações clínicas variadas desde o período neonatal por acometer vários órgãos ou sistemas. $\mathrm{Na}$ época da ocorrência desse caso (2004), a triagem neonatal para FC ainda não era realizada em nosso meio, o que poderia ter antecipado o diagnóstico da doença. O diagnóstico clínico inicial da criança foi Kwashiorkor, sendo propostas as hipóteses de origem primária ou secundária. A primária foi aventada pelo fato de que houve separação dos pais no final da gestação e isso poderia levar a uma hipogalactia por estresse emocional. Contudo, já na história havia sido tentada a suplementação sem sucesso, tornando essa hipótese pouco provável.

A alergia à proteína do leite de vaca é uma das causas de enteropatia perdedora de proteínas, mas não há relato conhecido de coagulopatia grave por deficiência de vitamina $\mathrm{K}$ nessa condição. Também, habitualmente, há melhora com hidrolisado proteico, o que não ocorreu no presente caso. A ocorrência de alergia à proteína do leite de vaca não é maior em pacientes com FC, em comparação à população em geral $^{(8)}$.

A vitamina K é uma vitamina lipossolúvel cuja absorção, no intestino delgado, depende de sais biliares e enzimas pancreáticas. Em pacientes com FC pode ocorrer uma deficiência dessa vitamina pela má absorção de gordura, que está presente em $80 \%$ dessas crianças. Além desse fator, outros podem contribuir como a doença hepática da fibrose cística, o uso prolongado de antibióticos ou quando há ressecção do intestino delgado por complicações gastrointestinais, como íleo meconial ${ }^{(9)}$. O distúrbio de coagulação como forma de apresentação da doença é raro, sendo descritos poucos casos na literatura ${ }^{(5,10)}$. No presente relato, o distúrbio de coagulação respondeu à administração da vitamina $\mathrm{K}$.

Rashid et al ${ }^{(11)}$ realizaram um estudo para diagnosticar a deficiência de vitamina K em 98 pacientes com FC (83 com insuficiência pancreática). Foi feita a dosagem da proteína induzida pela ausência da vitamina K (PIVKA-II) e $78 \%$ dos pacientes com insuficiência pancreática e todos aqueles com FC associada à doença hepática apresentaram níveis elevados de PIVKA-II ( $\geq 3 \mu \mathrm{g} / \mathrm{L}$ ), indicando a necessidade de uma suplementação de rotina. Nesses pacientes com alteração do PIVKA-II, seis apresentaram tempo de protrombina prolongado.

Para os lactentes com FC, os Consensos Americano e Europeu de Nutrição recomendam o aleitamento materno administrado em conjunto com enzimas pancreáticas, sendo a fonte de alimentação primária no primeiro ano de vida e a introdução da alimentação complementar entre quatro e seis meses. As fórmulas infantis apropriadas podem ser utilizadas na impossibilidade do aleitamento materno ou como complemento ${ }^{(8,12)}$. O aleitamento materno deve ser incentivado por auxiliar no desenvolvimento do sistema imune do lactente, fornecendo os componentes que apresentam atividade antimicrobiana, antiviral, antiinflamatória e de imunomodulação ${ }^{(13,14)}$. Quando a ingestão alimentar do paciente está abaixo das necessidades, estão indicados suplementos nutricionais orais e, não havendo resposta favorável, inicia-se a nutrição enteral na tentativa de melhorar o estado nutricional. O baixo ganho ponderal pode se dever tanto à redução da ingestão alimentar, quanto ao aumento do gasto energético ou a uma má absorção de nutrientes, apesar do uso de enzimas pancreáticas, ou ainda a uma combinação de fatores ${ }^{(4)}$.

A não realização do teste do suor por instabilidade do quadro clínico se justifica, mas não o argumento de que o exame é de pouco valor em crianças com anasarca. Yigit, Selimoglu e Altinkaynak ${ }^{(15)}$ realizaram o teste do suor em 90 crianças com desnutrição calórico proteica de origem primária, divididos equitativamente em $1^{\circ}, 2^{\circ}$ e $3^{\circ}$ graus de acordo com os critérios de Gomez et a ${ }^{(16)}$. O teste do suor mostrou alteração em apenas seis pacientes, todos com desnutrição de $3^{\circ}$ grau e nenhum valor excedeu $69 \mathrm{mmol} / \mathrm{L}$. Em um estudo de caso de FC relatado por Damaceno, Muramatu e Alonso ${ }^{(17)}$, o paciente apresentou níveis extremamente reduzidos de albumina sérica (1,53 e 1,40mg\%) e elevados de eletrólitos 
no suor (cloro 148 e $89 \mathrm{mEq} / \mathrm{L}$, sódio 124 e 83 Eq/L) em duas amostras realizadas em dias diferentes, mesmo com as condições clínicas de edema e desnutrição que poderiam ocasionar um resultado falso-negativo no exame.

As deficiências de enteroquinase e tripsinogênio são situações muito raras, nas quais os pacientes apresentam, principalmente, má digestão proteica. Embora raras, devem ser consideradas em toda criança com edema e hipoproteinemia, depois de descartadas as causas mais frequentes. Por outro lado, na síndrome de Shwachman-Diamond há uma insuficiência pancreática exócrina com diminuição acentuada de todas as enzimas pancreáticas. Nessa síndrome, também podem ocorrer outras alterações, como neutropenia e distúrbios ósseos.

A manifestação cutânea precoce na FC é rara e tem sido atribuída às deficiências nutricionais de zinco, proteínas e ácidos graxos essenciais. Em geral, as alterações cutâneas precedem o aparecimento de edema e outras manifestações clínicas típicas da doença ${ }^{(18)}$. Na literatura, existem relatos

\section{Referências bibliográficas}

1. Beaudet AL. Cystic fibrosis. In: Scriver CR, Beaudet AL, Sly WS, Valle D, editors. The metabolic basis of inherited disorders. $6^{\text {th }}$ ed. New York: McGrawHill; 1989. p.2649-80.

2. Reed TE. Caucasian genes in American Negroes. Science 1969;165:762-8.

3. Castro LV, Martins MC. Application of Schwachman's scoring system to patients with cystic fibrosis with focus on the nutritional aspect. Rev Pediatr Ceará 2005;6:27-36.

4. Reis FJ, Damaceno N. Cystic fibrosis. J Pediatr (Rio J) 1998;74 (Supl 1):S76-94.

5. Mérelle ME, Griffioen RW, Dankert-Roelse LE. Cystic fibrosis presenting with intracerebral haemorrhage. Lancet 2001;358:1960.

6. Mei-Zahav M, Solomon M, Kawamira A, Coates A, Durie P. Cystic fibrosis presenting as kwashiorkor in a Sri Lankan infant. Arch Dis Child 2003;88:724-5.

7. Tanner JM, Whitehouse RH. Clinical longitudinal standards for height, weight, height velocity, weight velocity, and stages of puberty. Arch Dis Child 1976;51:170-9.

8. Borowitz D, Baker RD, Stallings V. Consensus report on nutrition for pediatric patients with cystic fibrosis. J Pediatr Gastroenterol Nutr 2002;35:246-59.

9. Durie PR. Vitamin $\mathrm{K}$ and the management of patients with cystic fibrosis. CMAJ 1994;151:933-6.

10. Verghese T, Beverley D. Vitamin $\mathrm{K}$ deficient bleeding in cystic fibrosis. Arch Dis Child 2003;88:553. desta manifestação atípica que melhoraram com a reposição de zinco, enzimas pancreáticas e vitaminas lipossolúveis ${ }^{(19,20)}$. A deficiência de zinco é difícil de ser caracterizada, uma vez que pode estar presente, mesmo quando o nível de zinco plasmático está normal ${ }^{(8)}$. No presente caso, o nível sérico de zinco foi baixo e deve estar implicado na etiologia dessa dermatose.

O diagnóstico final foi de FC que, nesse caso, manifestouse de uma forma atípica. O Kwashiorkor como manifestação da FC é raro, mas já existem descrições na literatura e pode-se confundir com desnutrição primária em países em desenvolvimento e subdesenvolvidos ${ }^{(6,17)}$. No Kwashiorkor de origem primária pode ocorrer também fibrose pancreática, o que é raro e, quando ocorre, é de grau leve ${ }^{(21)}$. A necropsia foi importante para confirmar o diagnóstico.

Em conclusão, a FC pode se manifestar como Kwashiorkor e distúrbio de coagulação por deficiência de vitamina K, devendo os profissionais de saúde estar atentos a essa possibilidade etiológica no diagnóstico diferencial dessa situação.

11. Rashid M, Durie P, Andrew M, Kalnins D, Shin J, Corey M et al. Prevalence of vitamin K deficiency in cystic fibrosis. Am J Clin Nutr 1999;70:378-82.

12. Sinaasappel M, Stern M, Littlewood J, Wolfe S, Steinkamp G, Heijerman HG, et al. Nutrition in patients with cystic fibrosis: a European Consensus. J Cyst Fibros 2002;1:51-75.

13. Barros MD, Kulesza TM, Rañna W, Carneiro-Sampaio MM. Papel do leite materno na defesa do lactente contra infecções. Pediatria (S Paulo) 1982;4:88-102.

14. Field CJ. The immunological components of human milk and their effect on immune development in infants. J Nutr 2005;135:1-4.

15. Yigit $\mathrm{H}$, Selimoglu MA, Altinkaynak $\mathrm{S}$. Sweat test results in children with primary protein energy malnutrition. J Pediatr Gastoenterol Nutr 2003;37:242-5.

16. Gomez F, Galvan RR, Cravioto J, Frenk S. Malnutrition in infancy and childhood with special reference to kwashiorkor. Adv Pediatr 1955;7:131-69.

17. Damaceno N, Muramatu LH, Alonso FH. Kwashiorkor as early clinical manifestation in a baby with cystic fibrosis. J Pediatr (Rio J) 1994;70:243-6.

18. Bernstein ML, McCusker MM, Grant-Kels JM. Cutaneous manifestations of cystic fibrosis. Pediatr Dermatol 2008;25:150-7.

19. Wenk KS, Higgins KB, Greer KE. Cystic fibrosis presenting with dermatitis. Arch Dermatol 2010;146:171-4.

20. Gomes AP, Silva Filho LV, Reis VM. Manifestações cutâneas em pacientes com fibrose cística. An Bras Dermatol 1995;70:557-9.

21. Brooks SE, Golden MH. The exocrine pancreas in kwashiorkor and marasmus. Light and electron microscopy. West Indian Med J 1992;41:56-60. 